\title{
Quadratic D Stabilizable Satisfactory Fault-tolerant Control with Constraints of Consistent Indices for Satellite Attitude Control Systems
}

\author{
Han Xiaodong ${ }^{1}$ and Zhang Dengfeng ${ }^{2}$ \\ 1 Institute of Telecommunication Satellite, CAST, Beijing 100094 \\ ${ }^{2}$ Nanjing University of Science and Technology, Nanjing 210094,
}

P.R. China

\section{Introduction}

In the last twenty some years, much attention has been paid to the problem of fault-tolerant control in satellite attitude control systems and many methods have been developed and proven to be capable of tolerating certain types of system faults (see e.g., [1 5] and the references therein). However, these solutions focused mainly on keeping stability of the faulty systems and in less consideration of other performance indices. Actually, the performance requirements of practical satellite control systems are usually multi-objective even in faulty cases and it is desirable for fault tolerant systems to keep the required performance indices in a satisfactory and admissible region rather than the optimization of single index [6 7].

As is well known, in many practical applications, it is desirable to construct systems to achieve better transient property, strong anti-disturbance ability and adequate level of cost function performance. To this end, optimal controllers have been designed by assigning pole in a desired region (see e.g., [8] and [9]), using $\mathrm{H}_{\infty}$ norm-bound constraint on disturbance attenuation [10, 11] and the guaranteed cost control (see [12] and [13]), respectively. Unfortunately, few results have been considered such performance indices simultaneously. Meanwhile, once some components of satellite attitude control systems go wrong, it is difficult to confirm desired multiple performances by the existing fault-tolerant control. Thus, it is necessary to investigate the problem of fault-tolerant control with multiple performance constraints.

Therefore, it is our motivation to investigate the quadratic D stabilizable satisfactory faulttolerant control problem with consistent indices constraints for a class of satellite attitude control uncertain discrete-time systems subject to actuator failures. In view of possible actuator failure as well as uncertainties which do not satisfy matching conditions existing in both the state and control input matrices, we first derive the existence conditions of satisfactory fault-tolerant state-feedback control law. Then by LMI technique, a convex optimization problem is formulated to find the corresponding controller. The state-feedback controller is designed to guarantee the closed-loop system satisfying the pre-specified quadratic $\mathrm{D}$ stabilizability index, $\mathrm{H}_{\infty}$ norm-bound constraint on disturbance attenuation and having the quadratic cost performance simultaneously, for all admissible value-bounded 
uncertainties and possible actuator failures. Furthermore, the consistency of the performance indices mentioned earlier is discussed for fault-tolerant control. Finally, simulative example is provided to illustrate the validity of the proposed method and the necessity of such a satisfactory fault-tolerant control.

\section{Problem formulation}

The systems considered in this paper can be described as follows:

$$
\begin{aligned}
& \mathbf{x}(k+1)=(\mathbf{A}+\Delta \mathbf{A}) \mathbf{x}(k)+(\mathbf{B}+\Delta \mathbf{B}) \mathbf{u}^{f}(k)+\mathbf{D} \boldsymbol{\omega}(k) \\
& \mathbf{z}(k)=\mathbf{C} \mathbf{x}(k)+\mathbf{E} \mathbf{u}^{f}(k)
\end{aligned}
$$

where $\mathbf{x}(k) \in \mathfrak{R}^{n}$ is the state vector, $\mathbf{u}^{f}(k) \in \mathfrak{R}^{p}$ is the control input from the actuator that may be fault, $\mathbf{z}(k) \in \mathfrak{R}^{k}$ is the controlled output, $\boldsymbol{\omega}(k) \in \mathfrak{R}^{q}$ is the input disturbance and || $\boldsymbol{\omega}(k)||_{2} \leq \beta, \mathbf{A}, \mathbf{B}, \mathbf{C}$ and $\mathbf{D}$ are known real constant matrices with appropriate dimensions, $\Delta \mathbf{A}$ and $\Delta \mathbf{B}$ are unknown matrices representing parameter uncertainties in the state matrix and input matrix, respectively.

In much literature, time-varying matrices of uncertain parameters are assumed to be of the form $\left[\begin{array}{ll}\Delta \mathbf{A} & \Delta \mathbf{B}\end{array}\right]=\mathbf{H F}(k)\left[\mathbf{E}_{a} \mathbf{E}_{b}\right]$, where $\mathbf{H}, \mathbf{E}_{a}$ and $\mathbf{E}_{b}$ are known real constant matrices with appropriate dimensions, $\mathbf{F}(k)$ is an unknown real matrix satisfying $\mathbf{F}(k) \in \Omega:=\left\{\mathbf{F}(k) \in \mathfrak{R}^{i^{*} j} \mid \mathbf{F}^{T}(k) \mathbf{F}(k) \leq \mathbf{I}\right\}$. However, the uncertainty is often value bounded which is more universal and need not satisfy the so-called matching conditions in practical engineering. Thus, $\Delta \mathbf{A}$ and $\Delta \mathbf{B}$ denote value bounded uncertainties in this paper, i.e., ||$\Delta \mathbf{A}|| \leq a,|| \Delta \mathbf{B}|| \leq b$.

Suppose the states are available for state-feedback.

$$
\mathbf{u}(k)=\mathbf{K} \mathbf{x}(k)
$$

where $\mathbf{K} \in \mathfrak{R}^{p^{*} n}$ is the feedback gain matrix. For the control input, the following failure model in [18] is adopted for this study:

$$
\begin{gathered}
\mathbf{u}^{f}(k)=\mathbf{M u}(k) \\
\mathbf{M}=\operatorname{diag}\left[m_{1}, m_{2}, \cdots, m_{p}\right]
\end{gathered}
$$

where $\mathbf{M}$ denotes the actuator faults function matrix, $0 \leq m_{i l} \leq m_{i} \leq m_{i u}, m_{i l}<1, m_{i u} \geq 1$, $i=1,2, \cdots, p$.

Remark 1: In the above fault matrix $\mathbf{M}$, if $m_{i}=1$, it corresponds to the normal case $\mathbf{u}^{f}(k)=\mathbf{u}(k)$. If $m_{i}=0$, outage of actuator control signal occurs. If $0<m_{i l}<m_{i}<m_{i u}, m_{i l}<1$, $m_{i \mu} \geq 1$ and $m_{i} \neq 1$, the corresponding actuator would be in partial failure case. Hence, let $\mathbf{u}^{f}(k)$ denote the control input vector both in normal and actuator failures cases for faulttolerant control research in this paper.

The decomposition of fault function $\mathbf{M}$ is given below with a similar manner in [7], which will be used for our main results. Define

$$
\mathbf{M}_{0}=\operatorname{diag}\left[m_{01}, m_{02}, \cdots, m_{0 p}\right], \mathbf{J}=\operatorname{diag}\left[j_{1}, j_{2}, \cdots, j_{p}\right],|\mathbf{L}|=\operatorname{diag}\left[\left|l_{1}\right|,\left|l_{2}\right|, \cdots,\left|l_{p}\right|\right],
$$


where $m_{0 i}=\left(m_{i l}+m_{i u}\right) / 2, j_{i}=\left(m_{i u}-m_{i l}\right) /\left(m_{i u}+m_{i l}\right), l_{i}=\left(m_{i}-m_{0 i}\right) / m_{0 i}$. So, we then have

$$
\mathbf{M}=\mathbf{M}_{0}(\mathbf{I}+\mathbf{L}) \quad|\mathbf{L}| \leq \mathbf{J} \leq \mathbf{I}
$$

The faulty closed-loop system is given by

$$
\begin{aligned}
& \mathbf{x}(k+1)=\overline{\mathbf{A}}_{C} \mathbf{x}(k)+\mathbf{D} \boldsymbol{\omega}(k) \\
& \mathbf{z}(k)=\mathbf{C}_{C} \mathbf{x}(k)
\end{aligned}
$$

where $\overline{\mathbf{A}}_{C}=\mathbf{A}_{C}+\Delta \mathbf{A}_{C}, \mathbf{A}_{C}=\mathbf{A}+\mathbf{B M K}, \Delta \mathbf{A}_{C}=\Delta \mathbf{A}+\Delta \mathbf{B M K}, \mathbf{C}_{C}=\mathbf{C}+\mathbf{E M K}$.

The cost function associated with system (1) considered possible actuator faults (3) is

$$
J=\sum_{k=0}^{\infty} \mathbf{x}^{T}(k)\left(\mathbf{Q}+(\mathbf{M K})^{T} \mathbf{R}(\mathbf{M K})\right) x(k)
$$

where $\mathbf{Q}=\mathbf{Q}^{T}>0, \mathbf{R}=\mathbf{R}^{T}>0$ are given weighting matrices.

Definition 1: For system (1), if there exists state-feedback controller, such that the faulty closed-loop system (6) will meet the following indices constraints simultaneously,

a. The closed-loop system is quadratic D stabilizable with constraint $\Phi(q, r), \Phi(q, r)$ denotes the disc with centre $q+j 0$ and the radius $r$, where $r$ and $q$ are known constants with $|q|+r<1$.

b. The $\mathrm{H}_{\infty}$ norm of the closed-loop transfer function is strictly less than a given positive scalar $\gamma$,

c. The closed-loop value of the cost function (7) exists an upper bound satisfying $J \leq J^{*}$, then for all admissible uncertainties and possible faults, the given indices, quadratic D stabilizability index $\Phi(q, r), \mathrm{H}_{\infty}$ norm bound $\gamma>0$ and cost function performance $J^{*}>0$ are said to be consistent, state-feedback controller $\mathbf{u}(k)=\mathbf{K x}(k)$ is said to be satisfactory fault-tolerant controller.

Now, the satisfactory fault-tolerant control problem considered in this paper is stated in the following.

Problem: For the system (1) with actuator failure, given the quadratic D stabilizability index $\Phi(q, r), \mathrm{H}_{\infty}$ norm bound $\gamma>0$ and the cost function (7), determine a control law $\mathbf{u}(k)=\mathbf{K} \mathbf{x}(k)$ so that the closed-loop system satisfies criteria (a), (b) and (c) simultaneously.

\section{Main results}

Lemma 1: Consider the actuator fault model (3), for any matrix $\mathbf{R}=\mathbf{R}^{T}>0$ and scalar $\varepsilon>0$, if $\mathbf{R}^{-1}-\varepsilon \mathbf{I}>0$ then

$$
\mathbf{M R M} \leq \mathbf{M}_{0}\left(\mathbf{R}^{-1}-\varepsilon \mathbf{I}\right)^{-1} \mathbf{M}_{0}+\varepsilon^{-1} \mathbf{M}_{0} \mathbf{J J} \mathbf{M}_{0}
$$

Lemma 2: Consider the system (1) subject to faults, given index $\Phi(q, r)$, if there exists gain matrix $\mathbf{K}$ and symmetric positive matrix $\mathbf{P}$ such that the following matrix inequality

$$
\left[\begin{array}{cc}
-\mathbf{P}^{-1} & \overline{\mathbf{A}}_{C}-q \mathbf{I} \\
\left(\overline{\mathbf{A}}_{C}-q \mathbf{I}\right)^{T} & -r^{2} \mathbf{P}
\end{array}\right]<0
$$


holds for all admissible uncertainties and possible faults, then the system (1) is quadratically D stabilizable.

Remark 2: It can be easily shown that in the case when $q=0, r=1$, the definition of quadratic D stabilizability is reduced to quadratic stabilizability where no closed-loop pole constraints is considered. Therefore, Lemma 2 shows that if the uncertain system (1) is quadratic D stabilizable, then for some state feedback controllers both quadratic stabilizability and pole assignment constraints of the faulty closed-loop system are enforced simultaneously.

Theorem 1: Consider the system (1), for the given index $\Phi(q, r)$, if there exists symmetric positive matrix $\mathbf{X}$, matrix $\mathbf{Y}$ and scalars $\varepsilon_{i}>0(i=1 \sim 3)$ such that the following linear matrix inequality

$$
\left[\begin{array}{ccccc}
\Sigma_{11} & \mathrm{AX}+\mathrm{BY}-q \mathrm{X} & 0 & \varepsilon_{3} \mathrm{BJ} & 0 \\
* & -r^{2} \mathrm{X} & \mathrm{X} & \mathrm{Y}^{T} & \mathrm{Y}^{T} \\
* & * & -\varepsilon_{1} \mathrm{I} & 0 & 0 \\
* & * & * & -\varepsilon_{2} \mathrm{I}+\varepsilon_{3} \mathrm{~J} & 0 \\
* & * & * & * & -\varepsilon_{3} \mathrm{~J}^{-1}
\end{array}\right]<0
$$

holds, where $\Sigma_{11}=-\mathrm{X}+\varepsilon_{1} a \mathrm{I}+\varepsilon_{2} b \mathrm{I}+\varepsilon_{3} \mathrm{BJB}^{T}$. Then for all admissible uncertainties and possible faults $\mathbf{M}$, the faulty closed-loop system (6) with satisfactory fault-tolerant controller $\mathbf{u}(k)=\mathbf{K} \mathbf{x}(k)=\mathbf{M}_{0}^{-1} \mathbf{Y} \mathbf{X}^{-1} \mathbf{x}(k)$ is quadratically D stabilizable.

Remark 3: Theorem 1 shows us LMI with $\mathbf{X}$ and $\mathbf{Y}$, which can be tested with convex optimization to decide whether it is solvable, and Matlab LMI Control Toolbox can be utilized to solve it. If LMI (13) holds, there must exist state-feedback controller assigning the closed-loop poles within $\Phi(q, r)$, namely, the constraint (a) is met. In this case the system (1) is said to be robust fault-tolerant state feedback assignable for actuator faults case.

Lemma 3: Consider the system (1) in fault case and the cost function (7) as well as square integrable disturbance $\boldsymbol{\omega}(k)$, if there exists gain matrix $\mathbf{K}$ and symmetric positive matrix $\mathbf{P}$ such that the following matrix inequality

$$
\overline{\mathbf{A}}_{C}^{T} \mathbf{P} \overline{\mathbf{A}}_{C}-\mathbf{P}+\mathbf{C}_{C}^{T} \mathbf{C}_{C}+\mathbf{Q}+\mathbf{K}^{T} \mathbf{M} \mathbf{R} \mathbf{M}+\overline{\mathbf{A}}_{C}^{T} \mathbf{P D}\left(\gamma^{2} \mathbf{I}-\mathbf{D}^{T} \mathbf{P D}\right)^{-1} \mathbf{D}^{T} \mathbf{P} \overline{\mathbf{A}}_{C}<0
$$

holds for all admissible uncertainties and possible faults $\mathbf{M}$, then the faulty closed-loop system is asymptotically stable with an $\mathrm{H}_{\infty}$ norm-bound $\gamma$, and the cost function (7) has an upper bound

$$
J<\mathbf{x}_{0}^{T} \mathbf{P} \mathbf{x}_{0}+\gamma^{2} \beta^{2}
$$

Remark 4: In some literature on the guaranteed cost control with regional pole constraint such as [12], the upper bound of cost function $J$ is that $J \leq V(0) / r^{2}=\mathbf{x}_{0}^{T} \mathbf{P} \mathbf{x}_{0} / r^{2}$. For $0<r<1$, it is certainly larger than the one in (12) when $\boldsymbol{\omega}(t)=0$. So the result here provides an improved performance bound.

Remark 5: Note this upper bound in (12) which depends on the initial condition $\mathbf{x}_{0}$. To remove the dependence on the initial state, suppose $\mathbf{x}_{0}$ is arbitrary but belongs to the set $W=\left\{\mathbf{x}_{0} \in \mathfrak{R}^{n}: \mathbf{x}_{0}=\mathbf{U v}, \mathbf{v}^{T} \mathbf{v} \leq 1\right\}$, where $\mathbf{U}$ is a given matrix. The cost bound in (12) then leads to 


$$
J<\mathbf{x}_{0}^{T} \mathbf{P} \mathbf{x}_{0}+\gamma^{2} \beta^{2} \leq \lambda_{\max }\left(\mathbf{U}^{T} \mathbf{P U}\right)+\gamma^{2} \beta^{2}
$$

Theorem 2: Consider the system (1) and the cost function (7), for the given index $\Phi(q, r)$ and $\mathrm{H}_{\infty}$ norm-bound index $\gamma$, if there exists symmetric positive matrix $\mathbf{X}$, matrix $\mathbf{Y}$ and scalars $\varepsilon_{i}>0(i=4 \sim 9)$ such that the following linear matrix inequality

$$
\left[\begin{array}{cccc}
-\mathrm{X} & 0 & (\mathrm{AX}+\mathrm{BY})^{T} & \Sigma_{21} \\
* & -\gamma^{2} \mathrm{I} & \mathrm{D}^{T} & 0 \\
* & * & -\mathrm{X}+\varepsilon_{4} a \mathrm{I}+\varepsilon_{5} b \mathrm{I}+\varepsilon_{6} \mathrm{BJB}^{T} & 0 \\
* & * & * & \Sigma_{22}
\end{array}\right]<0
$$

holds, where $\Sigma_{21}=\left[(\mathrm{CX}+\mathrm{EY})^{T}, \mathrm{X}, \mathrm{X}, \mathrm{Y}^{T}, \mathrm{Y}^{T}, \mathrm{Y}^{T}, \mathrm{Y}^{T}, \mathrm{Y}^{T}, \mathrm{Y}^{T} \mathrm{~J}\right], \Sigma_{22}=\operatorname{diag}\left[-\mathrm{I}+\varepsilon_{7} \mathrm{EJE}^{T},-\mathrm{Q}^{-1},-\varepsilon_{4} \mathrm{I}\right.$, $\left.-\varepsilon_{5} \mathrm{I}+\varepsilon_{8} \mathrm{~J},-\varepsilon_{6} \mathrm{~J}^{-1},-\varepsilon_{7} \mathrm{~J}^{-1},-\varepsilon_{8} \mathrm{~J}^{-1}, \varepsilon_{9} \mathrm{I}-\mathrm{R}^{-1},-\varepsilon_{9} \mathrm{I}\right]$. Then for all admissible uncertainties and possible faults $\mathbf{M}$, the faulty closed-loop system (6) with satisfactory fault-tolerant controller $\mathbf{u}(k)=\mathbf{K x}(k)=\mathbf{M}_{0}^{-1} \mathbf{Y} \mathbf{X}^{-1} \mathbf{x}(k)$ is asymptotically stable with an $\mathrm{H}_{\infty}$ norm-bound $\gamma$, and the corresponding closed-loop cost function (7) is with $J \leq \lambda_{\max }\left(\mathbf{U}^{T} \mathbf{X}^{-1} \mathbf{U}\right)+\gamma^{2} \beta^{2}$.

According to Theorem 1 and 2, the consistency of the quadratic D stabilizability constraint, $\mathrm{H}_{\infty}$ performance and cost function indices for fault-tolerant control is deduced as the following optimization problem.

Theorem 3: Given quadratic D stabilizability index $\Phi(q, r)$, suppose the system (1) is robust fault-tolerant state feedback assignable for actuator faults case, then LMIs (10), (13) have a feasible solution. Thus, the following minimization problem is meaningful.

$$
\min (\gamma):\left(\mathbf{X}, \mathbf{Y}, \gamma, \varepsilon_{i}\right) \text { S.t. LMIs (10), (13) }
$$

Proof: Based on Theorem 1, if the system (1) is robust fault-tolerant state feedback assignable for actuator faults case, then inequality

$$
\overline{\mathbf{A}}_{C}^{T} \mathbf{P} \overline{\mathbf{A}}_{C}-\mathbf{P}<0
$$

has a feasible solution $\mathbf{P}, \mathbf{K}$. And existing $\lambda>0, \delta>0$, the following inequality holds

$$
\lambda\left[\overline{\mathbf{A}}_{C}^{T} \mathbf{P} \overline{\mathbf{A}}_{C}-\mathbf{P}\right]+\mathbf{C}_{C}{ }^{T} \mathbf{C}_{C}+\mathbf{Q}+\mathbf{K}^{T} \mathbf{M R M K}+\delta \mathbf{I}<0
$$

Then existing a scalar $\gamma_{0}$, when $\gamma>\gamma_{0}$, it can be obtained that

$$
\overline{\mathbf{A}}_{C}^{T} \mathbf{P}_{1} \mathbf{D}\left(\gamma^{2} \mathbf{I}-\mathbf{D}^{T} \mathbf{P}_{1} \mathbf{D}\right)^{-1} \mathbf{D}^{T} \mathbf{P}_{1} \overline{\mathbf{A}}_{C}<\delta \mathbf{I}
$$

where $\mathbf{P}_{1}=\lambda \mathbf{P}$. Furthermore, it follows that

$$
\overline{\mathbf{A}}_{C}^{T} \mathbf{P}_{1} \overline{\mathbf{A}}_{C}-\mathbf{P}_{1}+\mathbf{C}_{C}{ }^{T} \mathbf{C}_{C}+\mathbf{Q}+\mathbf{K}^{T} \mathbf{M} \mathbf{R} \mathbf{M}+\overline{\mathbf{A}}_{C}^{T} \mathbf{P}_{1} \mathbf{D}\left(\gamma^{2} \mathbf{I}-\mathbf{D}^{T} \mathbf{P}_{1} \mathbf{D}\right)^{-1} \mathbf{D}^{T} \mathbf{P}_{1} \overline{\mathbf{A}}_{C}<0
$$

Using Schur complement and Theorem 2, it is easy to show that the above inequality is equivalent to linear matrix inequality (13), namely, $\mathbf{P}_{1}, \mathbf{K}, \gamma$ is a feasible solution of LMIs 
(10), (13). So if the system (1) is robust fault-tolerant state feedback assignable for actuator faults case, the LMIs (10), (13) have a feasible solution and the minimization problem (14) is meaningful. The proof is completed.

Suppose the above minimization problem has a solution $\mathbf{X}_{L}, \mathbf{Y}_{L}, \varepsilon_{i L}, \gamma_{L}$, and then any index $\gamma>\gamma_{L}$, LMIs (10), (13) have a feasible solution. Thus, the following optimization problem is meaningful.

Theorem 4: Consider the system (1) and the cost function (7), for the given quadratic D stabilizability index $\Phi(q, r)$ and $H_{\infty}$ norm-bound index $\gamma>\gamma_{L}$, if there exists symmetric positive matrix $\mathbf{X}$, matrix $\mathbf{Y}$ and scalars $\varepsilon_{i}>0(i=1 \sim 9)$ such that the following minimization

$$
\min \lambda+\gamma^{2} \beta^{2}
$$

$$
\begin{aligned}
\text { S.t. (i) } & (10),(13) \\
\text { (ii) } & {\left[\begin{array}{cc}
-\lambda \mathbf{I} & \mathbf{U}^{T} \\
\mathbf{U} & -\mathbf{X}
\end{array}\right]<0 }
\end{aligned}
$$

has a solution $\mathbf{X}_{\min }, \mathbf{Y}_{\min }, \varepsilon_{i \min }, \lambda_{\min }$, then for all admissible uncertainties and possible faults $\mathbf{M}, \mathbf{u}(k)=\mathbf{K x}(k)=\mathbf{M}_{0}^{-1} \mathbf{Y}_{\min } \mathbf{X}_{\min }^{-1} \mathbf{x}(k)$ is an optimal guaranteed cost satisfactory fault-tolerant controller, so that the faulty closed-loop system (6) is quadratically D stabilizable with an $\mathrm{H}_{\infty}$ norm-bound $\gamma$, and the corresponding closed-loop cost function (7) satisfies $J \leq \lambda_{\min }+\gamma^{2} \beta^{2}$.

According to Theorem $1 \sim 4$, the following satisfactory fault-tolerant controller design method is concluded for the actuator faults case.

Theorem 5: Given consistent quadratic D stabilizability index $\Phi(q, r), \mathrm{H}_{\infty}$ norm index $\gamma>\gamma_{L}$ and cost function index $J^{*}>\lambda_{\text {min }}+\gamma^{2} \beta^{2}$, suppose that the system (1) is robust faulttolerant state feedback assignable for actuator faults case. If LMIs (10), (13) have a feasible solution $\mathbf{X}, \mathbf{Y}$, then for all admissible uncertainties and possible faults $\mathbf{M}$, $\mathbf{u}(k)=\mathbf{K} \mathbf{x}(k)=\mathbf{M}_{0}^{-1} \mathbf{Y} \mathbf{X}^{-1} \mathbf{x}(k)$ is satisfactory fault-tolerant controller making the faulty closedloop system (6) satisfying the constraints (a), (b) and (c) simultaneously.

In a similar manner to the Theorem 5, as for the system (1) with quadratic D stabilizability, $\mathrm{H}_{\infty}$ norm and cost function requirements in normal case, i.e., $\mathbf{M}=\mathbf{I}$, we can get the satisfactory normal controller without fault tolerance.

\section{Simulative example}

Consider a satellite attitude control uncertain discrete-time system (1) with parameters as follows:

$$
\mathbf{A}=\left[\begin{array}{cc}
-2 & 2 \\
2 & 4
\end{array}\right], \mathbf{B}=\left[\begin{array}{ll}
1 & 1 \\
0 & 1
\end{array}\right], \mathbf{C}=\left[\begin{array}{ll}
0.2 & 0.3
\end{array}\right], \mathbf{D}=\left[\begin{array}{l}
0.1 \\
0.5
\end{array}\right], \mathbf{Q}=\left[\begin{array}{ll}
1 & 0 \\
0 & 1
\end{array}\right], \mathbf{R}=\left[\begin{array}{ll}
1 & 0 \\
0 & 1
\end{array}\right], a=0.1, b=0.2
$$

Suppose the actuator failure parameters $\mathbf{M}_{l}=\operatorname{diag}\{0.4,0.6\}, \mathbf{M}_{u}=\operatorname{diag}\{1.3,1.1\}$. Given the quadratic D stabilizability index $\Phi(0.5,0.5)$, we can obtain state-feedback satisfactory faulttolerant controller (SFTC), such that the closed-loop systems will meet given indices constraints simultaneously based on Theorem 5 . 


$$
\mathbf{K}_{\text {SFTC }}=\left[\begin{array}{cc}
0.5935 & 3.0187 \\
-6.7827 & -5.6741
\end{array}\right]
$$

In order to compare, we can obtain the state-feedback satisfactory normal controller (SNC) without fault-tolerance.

$$
\mathbf{K}_{\mathrm{SNC}}=\left[\begin{array}{cc}
0.4632 & 2.4951 \\
-5.4682 & -4.9128
\end{array}\right]
$$

Through simulative calculation, the pole-distribution of the closed-loop system by satisfactory fault-tolerant controller and normal controller are illustrated in Figure 1, 2 and 3 for normal case and the actuator faults case respectively. It can be concluded that the poles of closed-loop system driven by normal controller lie in the circular disk $\Phi(0.5,0.5)$ for normal case (see Fig. 1). However, in the actuator failure case, the closed-loop system with normal controller is unstable; some poles are out of the given circular disk (see Fig. 2). In the contrast, the performance by satisfactory fault-tolerant controller still satisfies the given pole index (see Fig. 3). Thus the poles of closed-loop systems lie in the given circular disk by the proposed method.

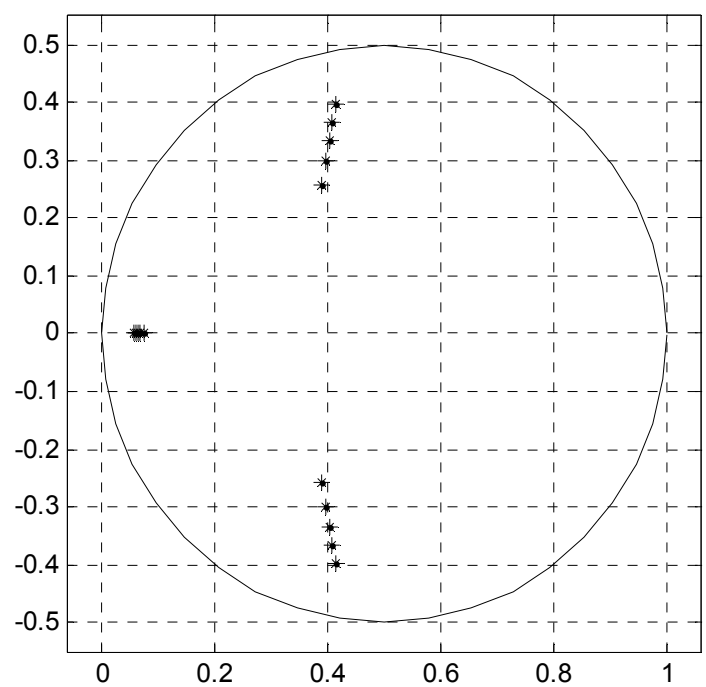

Fig. 1. Pole-distribution under satisfactory normal control without faults

\section{Conclusion}

Taking the guaranteed cost control in practical systems into account, the problem of satisfactory fault-tolerant controller design with quadratic $\mathrm{D}$ stabilizability and $\mathrm{H}_{\infty}$ normbound constraints is concerned by LMI approach for a class of satellite attitude systems subject to actuator failures. Attention has been paid to the design of state-feedback controller that guarantees, for all admissible value-bounded uncertainties existing in both the state and control input matrices as well as possible actuator failures, the closed-loop system to satisfy 
the pre-specified quadratic D stabilizability index, meanwhile the $\mathrm{H}_{\infty}$ index and cost function are restricted within the chosen upper bounds. So, the resulting closed-loop system can provide satisfactory stability, transient property, $\mathrm{H}_{\infty}$ performance and quadratic cost performance despite of possible actuator faults. The similar design method can be extended to sensor failures case.

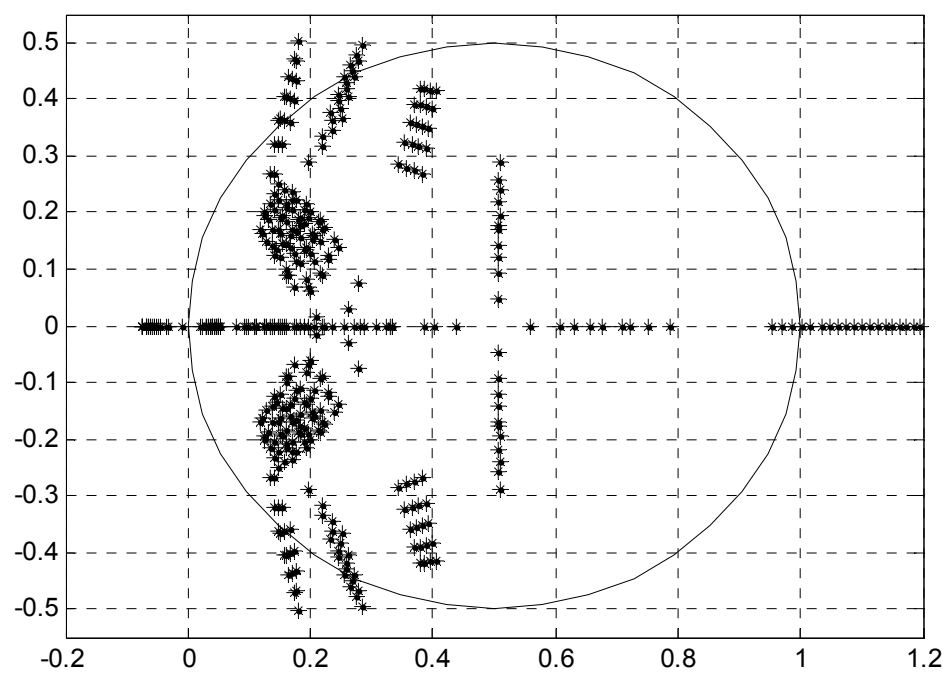

Fig. 2. Pole-distribution under satisfactory normal control with faults

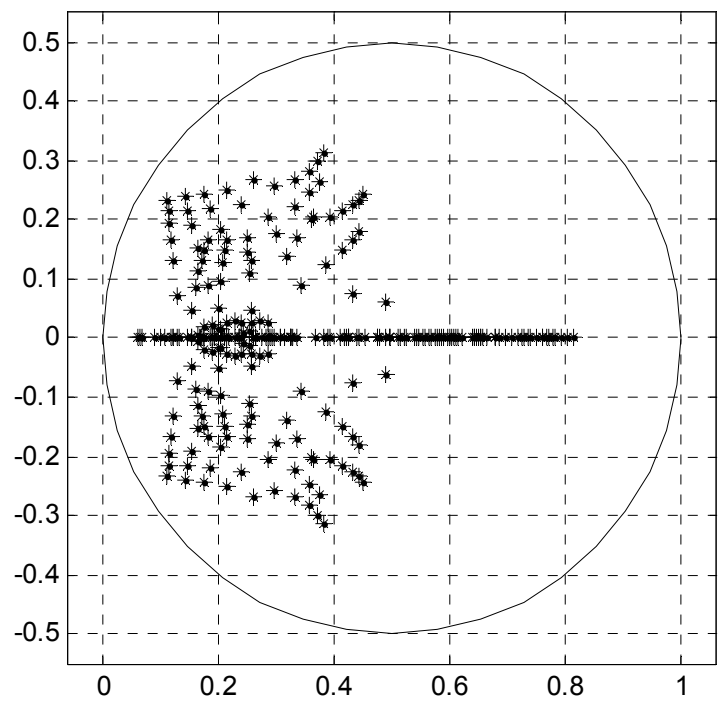

Fig. 3. Pole-distribution under satisfactory fault-tolerant control with faults 


\section{Acknowledgement}

This work is supported by the National Natural Science Foundation of P. R. China under grants 60574082, 60804027 and the NUST Research Funding under Grant 2010ZYTS012.

\section{Reference}

H. Yang, B. Jiang, and M. Staroswiecki. Observer-based fault-tolerant control for a class of switched nonlinear systems, IET Control Theory Appl, Vol. 1, No. 5, pp. 1523-1532, 2007.

D. Ye, and G. Yang. Adaptive fault-tolerant tracking control against actuator faults with application to flight control, IEEE Trans. on Control Systems Technology, Vol. 14, No. 6, pp. 1088-1096, 2006.

J. Lunze, and T. Steffen. Control reconfiguration after actuator failures using disturbance decoupling methods, IEEE Trans. on Automatic Control, Vol. 51, No. 10, pp. 15901601, 2006.

Y. Wang, D. Zhou, and F. Gao. Iterative learning fault-tolerant control for batch processes, Industrial \& Engineering Chemistry Research, Vol. 45, pp. 9050-9060, 2006.

M. Zhong, H. Ye, S. Ding, et al. Observer-based fast rate fault detection for a class of multirate sampled-data systems, IEEE Trans. on Automatic Control, Vol. 52, No. 3, pp. 520-525, 2007.

G. Zhang, Z. Wang, X. Han, et al. Research on satisfactory control theory and its application in fault-tolerant technology, Proceedings of the 5th World Congress on Intelligent Control and Automation, Hangzhou China, June 2004, Vol. 2, pp. 1521-1524.

D. Zhang, Z. Wang, and S. Hu. Robust satisfactory fault-tolerant control of uncertain linear discrete-time systems: an LMI approach, International Journal of Systems Science, Vol. 38, No. 2, pp. 151-165, 2007.

F. Wang, B. Yao, and S. Zhang. Reliable control of regional stabilizability for linear systems, Control Theory \& Applications, Vol. 21, No. 5, pp. 835-839, 2004.

F. Yang, M. Gani, and D. Henrion. Fixed-order robust $\mathrm{H}_{\infty}$ controller design with regional pole assignment, IEEE Trans. on Automatic Control, Vol. 52, No. 10, pp. 1959-1963, 2007.

A. Zhang, and $\mathrm{H}$. Fang. Reliable $\mathrm{H}_{\infty}$ control for nonlinear systems based on fuzzy control switching, Proceedings of the 2007 IEEE International Conference on Mechatronics and Automation, Harbin China, Aug. 2007, pp. 2587-2591.

F. Yang, Z. Wang, D. W.C.Ho, et al. Robust $\mathrm{H}_{\infty}$ control with missing measurements and time delays, IEEE Trans. on Automatic Control, Vol. 52, No. 9, pp. 1666-1672, 2007.

G. Garcia. Quadratic guaranteed cost and disc pole location control for discrete-time uncertain systems, IEE Proceedings: Control Theory and Applications, Vol. 144, No. 6, pp. 545-548, 1997.

X. Nian, and J. Feng. Guaranteed-cost control of a linear uncertain system with multiple time-varying delays: an LMI approach, IEE Proceedings: Control Theory and Applications, Vol. 150, No. 1, pp. 17-22, 2003.

J. Liu, J. Wang, and G. Yang. Reliable robust minimum variance filtering with sensor failures, Proceeding of the 2001 American Control Conference, Arlington USA, Vol. 2, pp. 1041-1046. 
H. Wang, J. Lam, S. Xu, et al. Robust $\mathrm{H}_{\infty}$ reliable control for a class of uncertain neutral delay systems, International Journal of Systems Science, Vol. 33, pp. 611-622, 2002.

G. Yang, J. Wang, and Y. Soh. Reliable $\mathrm{H}_{\infty}$ controller design for linear systems, Automatica, Vol. 37, pp. 717-725, 2001.

Q. Ma, and C. Hu. An effective evolutionary approach to mixed $\mathrm{H}_{2} / \mathrm{H}_{\infty}$ filtering with regional pole assignment, Proceedings of the 6th World Congress on Intelligent Control and Automation, Dalian China, June 2006, Vol. 2, pp. 1590-1593.

Y. Yang, G. Yang, and Y. Soh. Reliable control of discrete-time systems with actuator failures, IEE Proceedings: Control Theory and Applications, Vol. 147, No. 4, pp. 428-432, 2000.

L. Yu. An LMI approach to reliable guaranteed cost control of discrete-time systems with actuator failure, Applied Mathematics and Computation, Vol. 162, pp. 1325-1331, 2005.

L. Xie. Output feedback $\mathrm{H}_{\infty}$ control of systems with parameter uncertainty, International Journal of Control, Vol. 63, No. 4, pp. 741-750, 1996. 


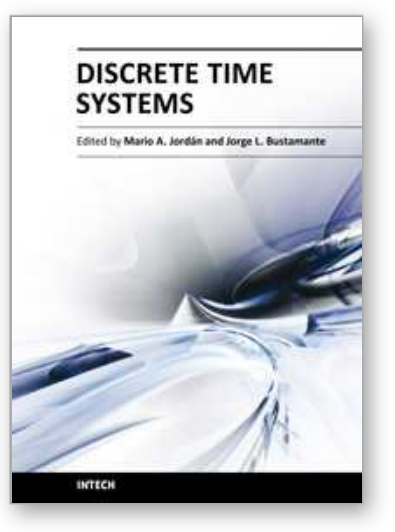

\author{
Discrete Time Systems \\ Edited by Dr. Mario Alberto Jord $\tilde{A}_{i n}$
}

ISBN 978-953-307-200-5

Hard cover, 526 pages

Publisher InTech

Published online 26, April, 2011

Published in print edition April, 2011

Discrete-Time Systems comprehend an important and broad research field. The consolidation of digital-based computational means in the present, pushes a technological tool into the field with a tremendous impact in areas like Control, Signal Processing, Communications, System Modelling and related Applications. This book attempts to give a scope in the wide area of Discrete-Time Systems. Their contents are grouped conveniently in sections according to significant areas, namely Filtering, Fixed and Adaptive Control Systems, Stability Problems and Miscellaneous Applications. We think that the contribution of the book enlarges the field of the Discrete-Time Systems with signification in the present state-of-the-art. Despite the vertiginous advance in the field, we also believe that the topics described here allow us also to look through some main tendencies in the next years in the research area.

\title{
How to reference
}

In order to correctly reference this scholarly work, feel free to copy and paste the following:

Han Xiaodong and Zhang Dengfeng (2011). Quadratic D Stabilizable Satisfactory Fault-tolerant Control with Constraints of Consistent Indices for Satellite Attitude Control Systems, Discrete Time Systems, Dr. Mario Alberto JordÃ $i n$ (Ed.), ISBN: 978-953-307-200-5, InTech, Available from:

http://www.intechopen.com/books/discrete-time-systems/quadratic-d-stabilizable-satisfactory-fault-tolerantcontrol-with-constraints-of-consistent-indices-

\section{INTECH}

open science | open minds

\section{InTech Europe}

University Campus STeP Ri

Slavka Krautzeka 83/A

51000 Rijeka, Croatia

Phone: +385 (51) 770447

Fax: +385 (51) 686166

www.intechopen.com

\section{InTech China}

Unit 405, Office Block, Hotel Equatorial Shanghai

No.65, Yan An Road (West), Shanghai, 200040, China

中国上海市延安西路65号上海国际贵都大饭店办公楼 405 单元

Phone: +86-21-62489820

Fax: $+86-21-62489821$ 
(C) 2011 The Author(s). Licensee IntechOpen. This chapter is distributed under the terms of the Creative Commons Attribution-NonCommercialShareAlike-3.0 License, which permits use, distribution and reproduction for non-commercial purposes, provided the original is properly cited and derivative works building on this content are distributed under the same license. 